\title{
Simulating the optical performances of the ATHENA X-ray telescope optics
}

Sironi, G.; Della Monica Ferreira, D.; Spiga, D.; Knudsen, E. Bergbäck; Bavdaz, M.; Bianucci, G.; Christensen, F. E.; Ferreira, I.; Fioretti, V.; Jegers, A. S

Total number of authors:

22

Published in:

Space Telescopes and Instrumentation 2018: Ultraviolet to Gamma Ray

Link to article, DOI:

$10.1117 / 12.2315019$

Publication date:

2018

Document Version

Publisher's PDF, also known as Version of record

Link back to DTU Orbit

Citation (APA):

Sironi, G., Della Monica Ferreira, D., Spiga, D., Knudsen, E. B., Bavdaz, M., Bianucci, G., Christensen, F. E., Ferreira, I., Fioretti, V., Jegers, A. S., Lotti, S., Macculi, C., Marioni, F., Massahi, S., Molendi, S., Moretti, A., Pareschi, G., Shortt, B., Tagliaferri, G., ... Wille, E. (2018). Simulating the optical performances of the ATHENA X-ray telescope optics. In J-W. A. den Herder, S. Nikzad, \& K. Nakazawa (Eds.), Space Telescopes and Instrumentation 2018: Ultraviolet to Gamma Ray (Vol. 10699). [106993N] SPIE - International Society for Optical Engineering. https://doi.org/10.1117/12.2315019

\section{General rights}

Copyright and moral rights for the publications made accessible in the public portal are retained by the authors and/or other copyright owners and it is a condition of accessing publications that users recognise and abide by the legal requirements associated with these rights.

- Users may download and print one copy of any publication from the public portal for the purpose of private study or research.

- You may not further distribute the material or use it for any profit-making activity or commercial gain

- You may freely distribute the URL identifying the publication in the public portal 


\section{Simulating the optical performances of the ATHENA x-ray telescope optics}

Giorgia Sironi, Daniele Spiga, Desiree Della Monica Ferreira, Ivo Ferreira, Marcos Bavdaz, et al.

Giorgia Sironi, Daniele Spiga, Desiree Della Monica Ferreira, Ivo Ferreira, Marcos Bavdaz, Erik Bergback Knudsen, Giovanni Bianucci, Finn Erland Christensen, Maximilien Collon, Fabio Marioni, Giovanni Pareschi, Arne 'S Jagers, Bianca Salmaso, Brian Shortt, Gianpiero Tagliaferri, Giuseppe Vacanti, Paolo Conconi, Giuseppe Valsecchi, Niels J. Westergaard, Eric Wille, Sonny Massahi, "Simulating the optical performances of the ATHENA x-ray telescope optics," Proc. SPIE 10699, Space Telescopes and Instrumentation 2018: Ultraviolet to Gamma Ray, 106993N (20 August 2018); doi: $10.1117 / 12.2315019$

Event: SPIE Astronomical Telescopes + Instrumentation, 2018, Austin, Texas, United States 


\title{
Simulating the optical performances of the ATHENA X-ray telescope optics
}

\author{
G. Sironi ${ }^{a}$ D. Della Monica Ferreira, ${ }^{b}$ D. Spiga,${ }^{a, c}$ E. Bergbäck Knudsen,${ }^{b}$ M. Bavdaz ${ }^{d}$
} G. Bianucci, ${ }^{e}$ F. E. Christensen, ${ }^{b}$ I. Ferreira,${ }^{d}$ V. Fioretti,${ }^{f}$ A. S Jegers,${ }^{b}$ S. Lotti,${ }^{h}$ C. Macculi,${ }^{h}$ F. Marioni ${ }^{e}$ S. Massahi ${ }^{b}$ S. Molendi, ${ }^{g}$ A. Moretti,${ }^{a}$ G. Pareschi,,${ }^{a}$ B. Shortt,${ }^{d}$ G. Tagliaferri, ${ }^{a}$ G. Valsecchi, ${ }^{e}$ N.J. Westergaard,${ }^{b}$ E. Wille ${ }^{d}$

${ }^{a}$ INAF - Brera Astronomical Observatory, Via Bianchi 46, 23807 Merate, Italy

${ }^{b}$ DTU Space, Techn. Univ. of Denmark, Elektrovej b. 327, 2800 Kgs. Lyngby, Denmark ${ }^{c}$ SLAC National Accelerator Laboratory, 2575 Sand Hill Road, 94025 Menlo Park, USA

${ }^{d}$ European Space Agency, ESTEC, Keplerlaan 1, 2201 AZ Noordwjik, The Netherlands

${ }^{e}$ Media Lario s.r.l., loc. Pascolo, 23842 Bosisio Parini, Italy

INAF - IASF Bologna, Via Gobetti 101, 40129 Bologna, Italy

${ }^{9} \mathrm{INAF}$ - IASF Milano, Via Bassini 15, 20133 Milano, Italy

${ }^{h}$ INAF - IAPS, Via Fosso del Cavaliere 100, 00133 Roma, Italy

\begin{abstract}
The ATHENA (Advanced Telescope for High Energy Astrophysics) X-ray observatory is an ESA-selected L2 class mission. In the proposed configuration, the optical assembly has a diameter of $2.2 \mathrm{~m}$ with an effective area of $1.4 \mathrm{~m}^{2}$ at $1 \mathrm{keV}, 0.25 \mathrm{~m}^{2}$ at $6 \mathrm{keV}$, and requires an angular resolution of 5 arcsec. To meet the requirements of effective area and angular resolution, the technology of Silicon Pore Optics (SPO) was selected for the optics implementation. The ATHENA's optic assembly requires hundreds of SPOs mirror modules (MMs), obtained by stacking wedged and ribbed silicon wafer plates onto silicon mandrels to form the Wolter-I configuration. Different factors can contribute to limit the imaging performances of SPOs, such as i) diffraction through the pore apertures, ii) plate deformations due to fabrication errors and surface roughness, iii) alignment errors among plates in an MM, and iv) co-focality errors within the MMs assembly. In order to determine the fabrication and assembling tolerances, the impact of these contributions needs to be assessed prior to manufacturing. A set of simulation tools responding to this need was developed in the framework of the ESA-financed projects SIMPOSIuM and ASPHEA. In this paper, we present the performance simulation obtained for the recentlyproposed ATHENA configuration in terms of effective area, and we provide a simulation of the diffractive effects in a pair of SPO MMs. Finally, we present an updated sizing of magnetic diverter (a Halbach array) and the magnetic fields levels that can be reached in order to deviate the most energetic protons out of the detector field.
\end{abstract}

Keywords: ATHENA, Silicon Pore Optics, optical design, simulation, alignment, diffraction

\section{INTRODUCTION}

The ATHENA X-ray observatory ${ }^{1}$ is an ESA large mission selected in the Cosmic Vision plan. The mission is planned to be launched to L2 in 2028. The proposed optical module of ATHENA consists of a single X-ray telescope with an effective area of about $1.4 \mathrm{~m}^{2}$ at $1.0 \mathrm{keV}$ and $0.25 \mathrm{~m}^{2}$ at $6 \mathrm{keV}$, focal length of $12 \mathrm{~m}$, and angular resolution of 5 arcsec.

To realize such a large aperture X-ray telescope, a technology based on Silicon Pore Optics (SPO) was selected. SPOs were developed in ESTEC since 2004 and relies on shaping Silicon plates to the desired optical profile. ${ }^{3}$ In summary, the manufacturing process consists of stacking doubly-side polished silicon wafers that have been wedged and grooved. The adhesion of the plates is ensured by molecular Van der Waals interactions between

e-mail: giorgia.sironi@inaf.it, Telephone: +39-02-72320468

Space Telescopes and Instrumentation 2018: Ultraviolet to Gamma Ray, edited by Jan-Willem A. den Herder, Shouleh Nikzad, Kazuhiro Nakazawa, Proc. of SPIE Vol. 10699, 106993N · C 2018 SPIE CCC code: $0277-786 X / 18 / \$ 18 \cdot$ doi: $10.1117 / 12.2315019$ 

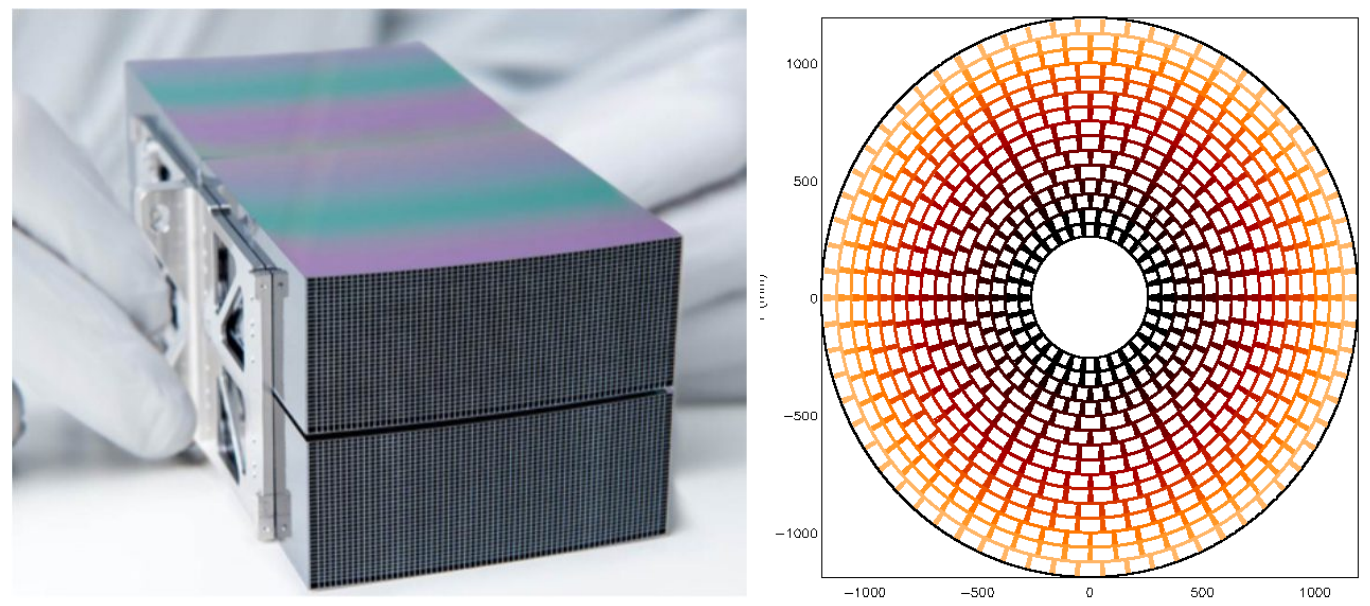

Figure 1. Left: A Wolter-I SPOs mirror module (image credits: ESA). The MM is realized as a double stack of 34 plates assembled in Wolter-I configuration. Right: Graphic representation of the MMs positions in the ATHENA telescope. The MMs are organized in 15 rows with hexagonal symmetry.

the two silicon oxide surfaces, when they come into contact. The shaping of the plates is defined by the first layer assembled, formed on a silicon mandrel with either parabolic (primary) or hyperbolic (secondary) shape, reconstructing, e.g., the widespread Wolter-I configuration. ${ }^{2}$ Each MM is obtained ${ }^{4}$ assembling the two shaped stacks, each composed by two sections of 34 layers: moreover, to simplify the final population of modules into the ATHENA optics structure, each MM includes two primary-secondary systems, operating in parallel. The typical height of a pore is of $0.606 \mathrm{~mm}$, and its width is $0.83 \mathrm{~mm}$ (Figure 1-left). Considering the silicon plate dimensions and the process characteristics, the MMs dimensional feasibility limit was set at about $100 \mathrm{~mm}$. This means that to populate the full telescope area a large number of MMs is required. In the configuration proposed by ESA (hereafter named configuration 3), the MMs are organized in 15 rows within six identical petals as in Figure 1-right, for a total of 678 units (Table 1).

The number of MMs to be produced is very large, but the SPOs technological solution offers great advantages for the realization of the optical module. They are:

- The replica process: just like nickel electroforming, the SPOs technology concentrates the efforts on the mandrel in order to improve the mirror quality. This means that time and costs for the shaping do not increase in proportion with the number of optical modules: they have to be allocated only once to produce the mandrel for each ring, instead of once per mirror like in the direct polishing technique. This solution has a strategic impact for mass production.

- The quality of the initial surfaces: just like thin glass foils, silicon plates are available on semiconductor markets at affordable costs with intrinsic low roughness. This avoids the iterative fabrication of superpolished optical surfaces required by direct polishing (for mirrors) and nickel electroforming (for mandrels).

- Physical characteristics of silicon: monocrystalline silicon has very high thermal conductivity, which makes it suitable to minimize the thermal gradients and the consequent thermal distortions.

The development of the SPOs technology also poses challenging aspects, which are being currently assessed:

- The approximation in the design: each plate in a MM does not come into contact with a mandrel with a designated shape when its shape is frozen into the stack: it is, rather replicated from the previous layer, with the addition of a wedge. Hence, it is not immediately ensured that the optical surfaces be representing the desired focusing optical shape. This approximation requires a considerable effort in terms of finite element analysis and a careful integration process in order to avoid accumulation of errors in the stack. 
Table 1. ATHENA's configuration 3 parameters. MMs are organized on 15 rows with hexagonal symmetry. The radius of the central plate is reported below. The width of the MMs was defined considering the symmetry and the feasibility limit of the plates. The length of the MMs was defined to make each module self-baffling.

\begin{tabular}{|c|c|c|c|c|}
\hline Row & \#MM & Radius $[\mathrm{m}]$ & Width $[\mathrm{mm}]$ & Lenght $[\mathrm{mm}]$ \\
\hline \hline 1 & 30 & 0.286 & 37.096 & 101.504 \\
\hline 2 & 30 & 0.348 & 50.158 & 83.388 \\
\hline 3 & 36 & 0.411 & 49.838 & 70.762 \\
\hline 4 & 42 & 0.473 & 49.613 & 61.46 \\
\hline 5 & 30 & 0.535 & 89.363 & 54.321 \\
\hline 6 & 36 & 0.597 & 82.476 & 48.671 \\
\hline 7 & 42 & 0.659 & 77.571 & 44.087 \\
\hline 8 & 42 & 0.722 & 86.892 & 40.294 \\
\hline 9 & 48 & 0.784 & 82.053 & 37.101 \\
\hline 10 & 48 & 0.846 & 90.205 & 34.383 \\
\hline 11 & 54 & 0.908 & 85.538 & 32.036 \\
\hline 12 & 54 & 0.970 & 92.782 & 29.99 \\
\hline 13 & 60 & 1.032 & 88.326 & 28.191 \\
\hline 14 & 60 & 1.095 & 94.845 & 26.597 \\
\hline 15 & 66 & 1.157 & 90.608 & 25.175 \\
\hline
\end{tabular}

- The effects on reflecting coating: in the originally proposed design, the baseline for the MMs coating was an $\mathrm{Ir} / \mathrm{B}_{4} \mathrm{C}$ bilayer, ${ }^{5}$ selected to optimize the effective area. Experimental tests of the coating performance performed at DTU Space have shown instability of the $\mathrm{B}_{4} \mathrm{C}$ coating, and incompatibility with the industrial processes involved in the production of SPO mirror modules. ${ }^{6}$ Silicon carbide ( $\mathrm{SiC}$ ) is recommended as replacement for $\mathrm{B}_{4} \mathrm{C}$. For the configuration 3 the proposed coating baseline is $\mathrm{Ir} / \mathrm{SiC} .{ }^{5,6}$

- The complex integration of the mirror assembly: considering the number of involved MMs, the misalignment contribution to be allocated in the error budget to the integration process should be carefully studied.

- The small size of pores: the pore structure generates a situation analogous to classical nested shell configuration, with reduced spacing in both directions (radial and azimuthal). This means that off-axis obstruction and stray-light modeling cannot be neglected. In addition, the pores are not so small to cause relevant diffraction effects in X-rays, but the diffraction contribution would be dominant in UV light. As UV optical benches are envisaged to perform the mirror module alignment in the assembly, the impact of aperture diffraction on the MMs alignment performances should be accurately assessed.

In this paper we discuss the latest results of a parallel set of studies, financed by ESA, to support the design and the development of the ATHENA optical module. SIMPOSiuM (SIMulation of Pore OpticS and Modeling) is a collaboration between the Brera Italian National Institute for Astrophysics (INAF-OAB) and the National Space Institute at the Technical University of Denmark (DTU Space) to develop software tools to simulate the optical performances of ATHENA. Results from the collaboration were already presented in previous papers. ${ }^{7,8}$ Here we report the latest results on the simulations of ATHENA configuration 3. ASPHEA (Alignment of Silicon Pore Optics for Height Energy Astrophysics) is a project led by Media Lario, involving INAF-OAB in the cofocality and diffraction simulation of MMs aligned on an UV bench. Results of this project were reported in previous works. ${ }^{7-9}$ Here we report the work obtained for the case of two MMs with alignment simulated at an X-ray test facility with source at finite distance, the PANTER facility of MPE. ${ }^{10}$ 

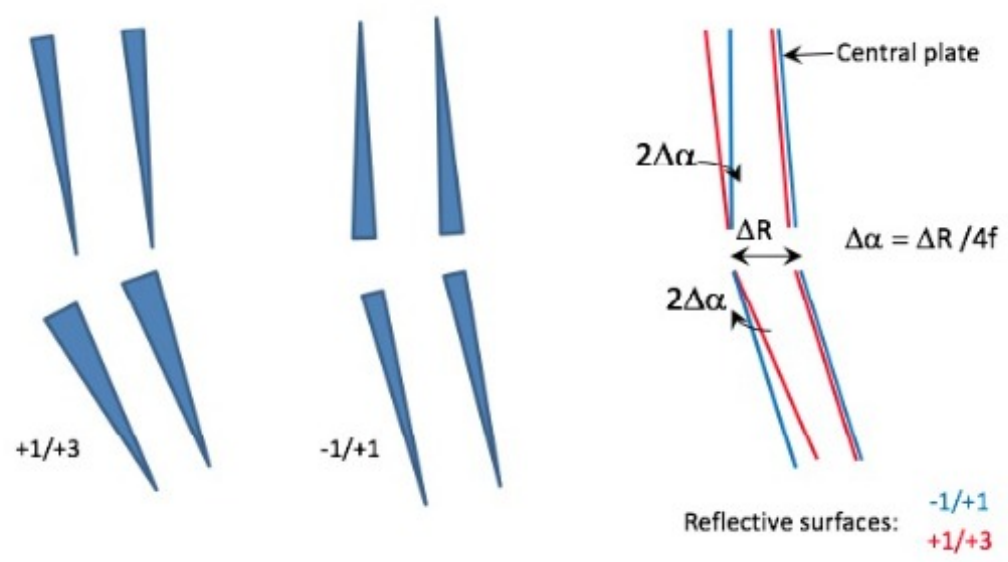

Figure 2. Different plate stacking in an SPO module, in radial section. Left: the $+1 /+3$ wedge configuration requires manufacturing two different kinds of wedged plates. Center: the $-1 /+1$ wedge configuration is obtained producing Silicon plates with the same wedge angle. Right: with respect to the $+1 /+3$ wedge, the angle variation in the $-1 /+1$ wedge configuration introduces an apparent source divergence $\delta n=2 n \Delta \alpha$ for the $\mathrm{n}$-th plate, counted from the central one.

\section{EFFECTIVE AREA SIMULATION}

The effective area of ATHENA can be calculated by means of the IDL code presented in previous papers. ${ }^{7,8}$ The code is based on the analytical computation of the illuminated area of each pore, extended to the full optical system. The code takes the following input information:

- Wolter or Wolter-Schwarzschild design: the intersection plane of different radially-distributed MMs is located at the same distance from the focal plane, along the axis or along the rays, respectively.

- $-1 /+1$ or $+1 /+3$ wedge configuration: in the Wolter-I design, the incidence angle of the rays on the reflecting surfaces should increase by $\Delta \alpha=\Delta R / 4 f$ on the primary mirror and by $3 \Delta \alpha$ on the secondary one. For faithfully representing the Wolter-I configuration, the primary stacks should have a wedge angle $\Delta \alpha$, while the secondary stacks should have a triple wedging $(+1 /+3)$. To simplify the manufacturing, however, a $-1 /+1$ configuration was proposed. This solution foresees the only use of $\Delta \alpha$-wedged plates mounted in opposite directions to maintain the nominal angular difference between the two reflecting surfaces (Fig. 2). The decrease of effective area due to a $+1 /-1$ wedge configuration is equivalent to a small off-axis effect, and was evaluated to be within $2 \%$. Such value is acceptable, when compared to the relevant process simplification enabled by a single wedge manufacturing throughout the entire stack. We note that, even if each MM is composed by two primary+secondary parallel stacks, each individual stack has its central plate at the nominal angle. This was accounted for in the computation and contributed to reduce the effective area loss to the mentioned $2 \%$ level.

- Variable filling factor $(F F)$ : ATHENA MMs are designed to be self-baffling. This means that the length of each MM is defined to make the maximum radius of a plate correspond to the inner radius of the next one $(\mathrm{FF}=1)$. This solution maximizes the effective area on-axis, but reduces the telescope field of view. In the case of SPOs technology, an approximation is intrinsic to the FF definition: all the plates composing a MM have the same length, but they are wedged to change the incidence angle, so it is possible to precisely assign the desired FF only to the central plate in each stack. Outer and inner plates would have FF $<1$ and $\mathrm{FF}>1$, respectively.

- Variable source off-axis angle: the code allows to compute the effective area also for off-axis sources. The off-axis angle increases the obscuration of the pores and causes a major effective area decrease.

In Figure 3, top panel, we report the effective area computed for ATHENA's configuration 3 (Tab. 1). The effective area computation was performed for Wolter I design, $-1 /+1$ wedging configuration and $\mathrm{FF}=1$. The 
computation was repeated for three cases of optimized reflecting coatings. ${ }^{6}$ In Fig. 3, central and bottom panels we report the results of the same computation for the cases of a 10 arcmin and 20 arcmin off-axis source. The effective area values obtained at $1 \mathrm{keV}$ and $6 \mathrm{keV}$ are reported in Tab. 2 for reference.
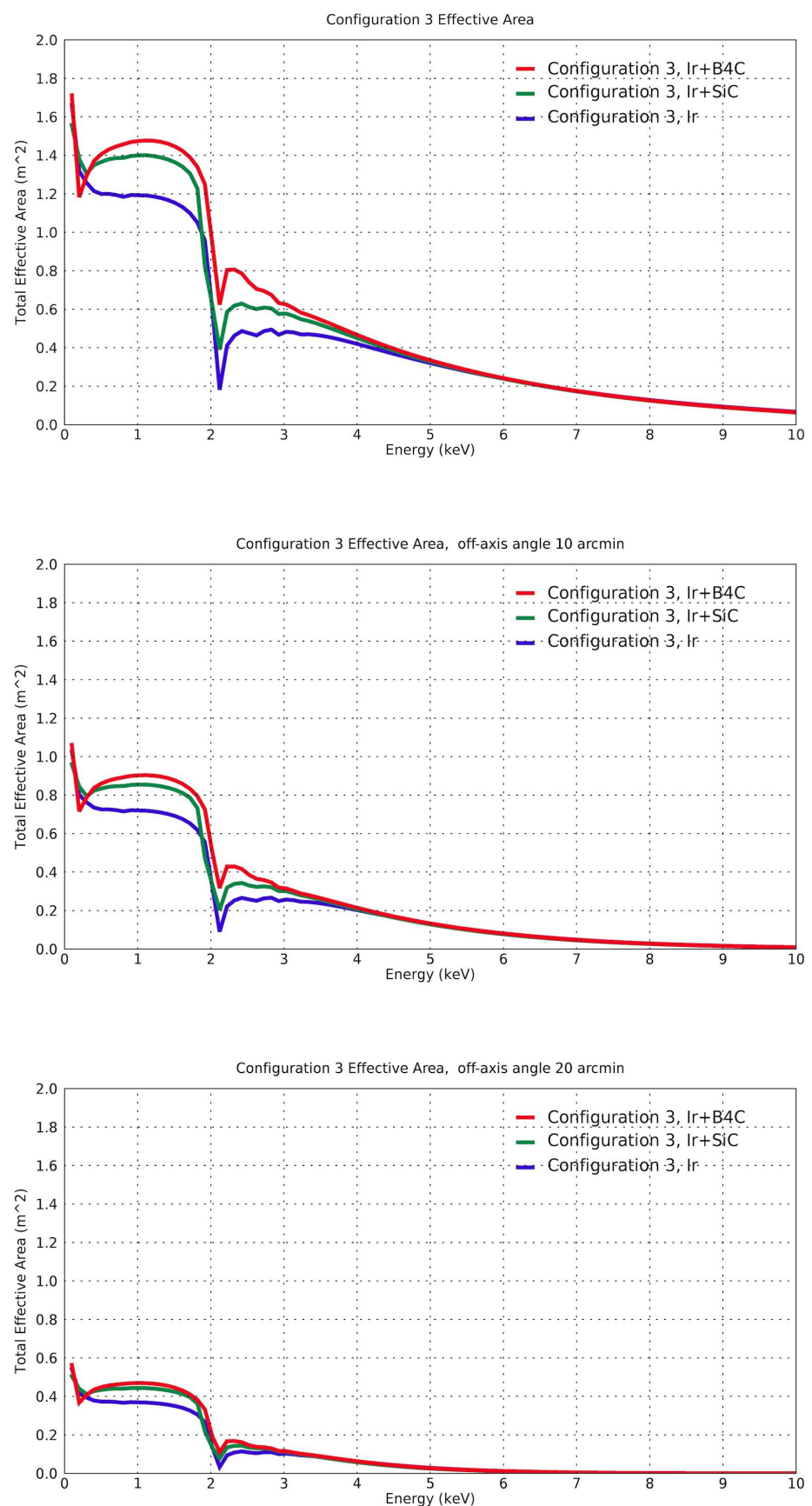

Figure 3. Energy-dependent Effective Area of ATHENA, configuration 3, as computed for three different coatings. The old configuration coating baseline $\mathrm{Ir} / \mathrm{B}_{4} \mathrm{C}$ (red curve), the new coating baseline $\mathrm{Ir} / \mathrm{SiC}$ (green curve), and a plain Ir coating (blue curve) are shown. Top panel: results for an on-axis source. Central panel: results for a 10 arcmin off-axis source. Bottom panel: results for a 20 arcmin off-axis source. 
Table 2. Effective areas computed for ATHENA configuration 3 at $1 \mathrm{keV}$ and $6 \mathrm{keV}$, for different off-axis angles.

\begin{tabular}{|l|c|c|c|c|c|c|}
\hline & \multicolumn{3}{|c|}{$A_{e f f} @ 1 \mathrm{keV}\left[\mathrm{m}^{2}\right]$} & \multicolumn{3}{c|}{$A_{e f f} @ 6 \mathrm{keV}\left[\mathrm{m}^{2}\right]$} \\
\hline Off-axis angle & 0 arcmin & 10 arcmin & 20 arcmin & 0 arcmin & 10 arcmin & 20 arcmin \\
\hline $\mathrm{Ir} / \mathrm{B} 4 \mathrm{C}$ & 1.474 & 0.902 & 0.469 & 0.242 & 0.080 & 0.011 \\
\hline $\mathrm{Ir} / \mathrm{SiC}$ & 1.399 & 0.854 & 0.443 & 0.238 & 0.077 & 0.010 \\
\hline $\mathrm{Ir}$ & 1.193 & 0.720 & 0.369 & 0.238 & 0.081 & 0.012 \\
\hline
\end{tabular}

\section{OPTICAL SIMULATION USING MCXTRACE}

Using an ATHENA-model setup with the ray tracing software package McXtrace, we may simulate various aspects of full-optic telescope performance. A simple way of doing this is to combine results from single mirror module simulations to obtain the performance of the entire optics.

Using McXtrace, we trace rays through the mirror pores to ultimately land to the focal point. As rays enter the mirror module pores and get reflected by the mirror coating, we are able to follow the rays and assess stray light, on- and off-axis effective area, PSF, among other effects.
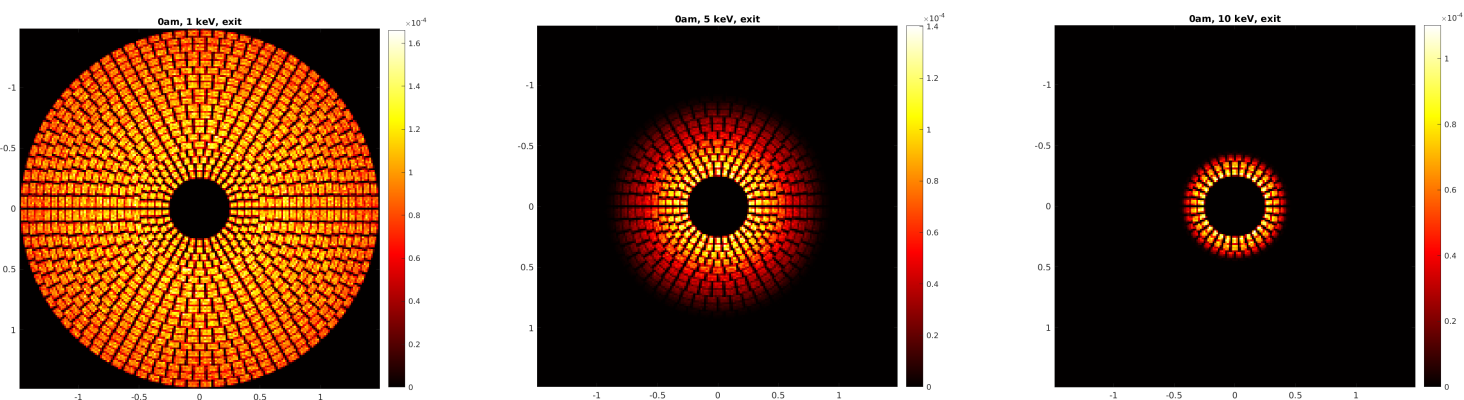

Figure 4. ATHENA optics through McXtrace ray-tracing. Example of simulated photons at exit of the optics for on-axis exposure with energies of $1 \mathrm{keV}$ (left), $5 \mathrm{keV}$ (center) and $10 \mathrm{keV}$ (right).
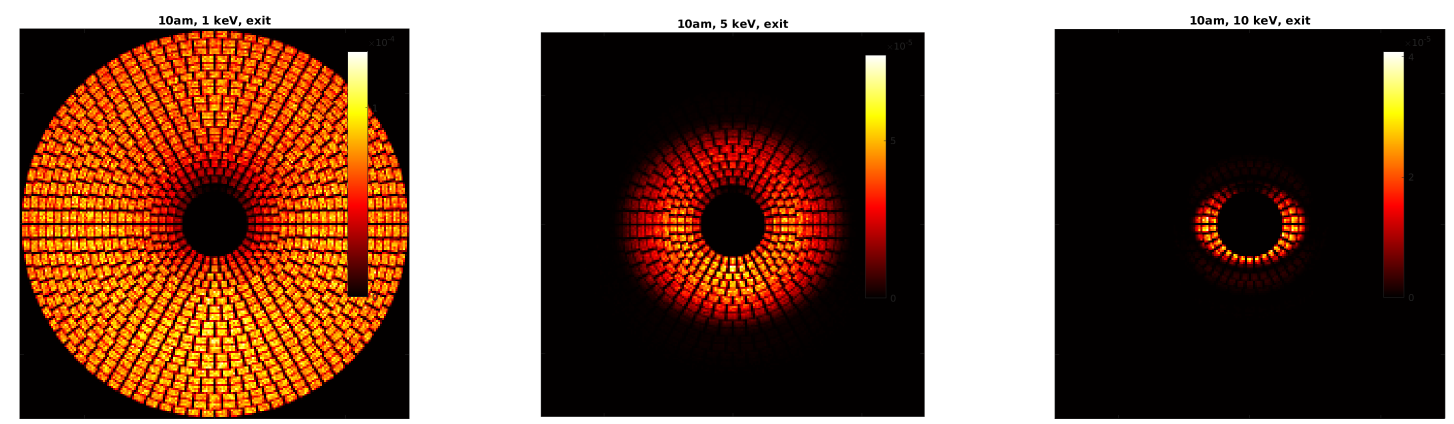

Figure 5. ATHENA optics through McXtrace ray-tracing. Example of simulated photons at exit of the optics for 10 arcmin off-axis exposure with energies of $1 \mathrm{keV}$ (left), $5 \mathrm{keV}$ (center) and $10 \mathrm{keV}$ (right).

The energy dependence of the optics performance is also evaluated as low energies, which will be reflected throughout the entire optics, while higher energies will lose reflectivity because of the higher grazing incidence angles on the outer mirror module rows.

Figure 4 shows the spatial intensity distribution just after the ATHENA optic for $1 \mathrm{keV}, 5 \mathrm{keV}$, and $10 \mathrm{keV}$ respectively. Here it is clear how the low energy rays are reflected by all mirror modules in all mirror module rows and how, as the X-ray energy increases, a smaller portion of the mirrors are able to reflect the traced rays. 
In much the same manner we can evaluate off-axis performance. Instead of having rays parallel to the optical axis impinge on the mirror modules, we let the rays come from an arbitrary angle. We show an example of the off-axis simulation in Fig. 5 for the case of 10 arcmin off-axis angle around the horizontal axis. Also here, the energy dependence in the reflection of rays is evident, illustrating the kind of losses that can be expected as a function of the X-ray energy and the off-axis angle.

\section{DIFFRACTION EFFECT OF MULTIPLE MIRROR MODULES}

Due to the large number of modules to be aligned, a non-negligible contribution to the angular resolution error budget has to be allocated to MMs integration. The geometrical misalignment of the MMs can be easily managed by McXtrace. However, the adoption of SPOs technology requires a deeper analysis of the diffraction effect due to the pore intrinsic high obstruction. This effect does not relevantly affect the X-ray angular resolution, which will be dominated by the plates shape errors, but it is quite strong in UV. Considering the case of the UV test facilities adopted for the MMs alignment during integration process, the simulation of diffractive effects is definitely relevant to ATHENA.

Usually, the effects of profile error and diffraction are faced with separate simulation tools: on one side, a geometrical ray-tracing is used to simulate the optical quality degradation due to surface shape error; on the other hand, the sole aperture diffraction can be simulated by means of the Fresnel integrals. The main problem in this approach is that the transition region between the two phenomena is extended, and a cut-off value of $\lambda$ is impossible to set in general. ${ }^{11}$

A conclusive solution is to simulate the whole system by means of physical optics. The treatment of the diffraction of SPOs and the introduced approximations were already been discussed in a previous paper. ${ }^{7} \mathrm{~A}$ direct comparison of the performed simulations with the experimental observed diffraction images was possible at Media Lario UV $(218 \mathrm{~nm})$ optical bench ${ }^{9}$ and provided a successful validation of the method. Finally, also the invariance of the PSF centroids positions of X-ray and UV images, even in presence of profiles longitudinal errors, was already presented one year ago. ${ }^{8}$ That confirmed that an integration process based on UV illumination is well suitable for SPOs to achieve the requested alignment accuracy.

Here, we show that the adoption of a light source of finite dimensions does not affect the alignment of two co-focal MMs. A simulation was performed modeling the Complex Pupil Function (CPF) of two flawless Wolter I MMs (Fig. 6). The MMs where represented as two $42 \mathrm{~mm}$ illuminated regions separated by a dark area of equal dimension. The radial distance of the central plate of the MMs is $737 \mathrm{~mm}$ and the pore dimensions are $0.606 \mathrm{~mm}$ by $0.83 \mathrm{~mm}$ with rib thickness of $0.17 \mathrm{~mm}$. The variable shade of red in Fig. 6 represent the optical path distance variation through the layers, increasing with the plate radius. This simulation reproduces a real alignment test performed at PANTER. ${ }^{9}$

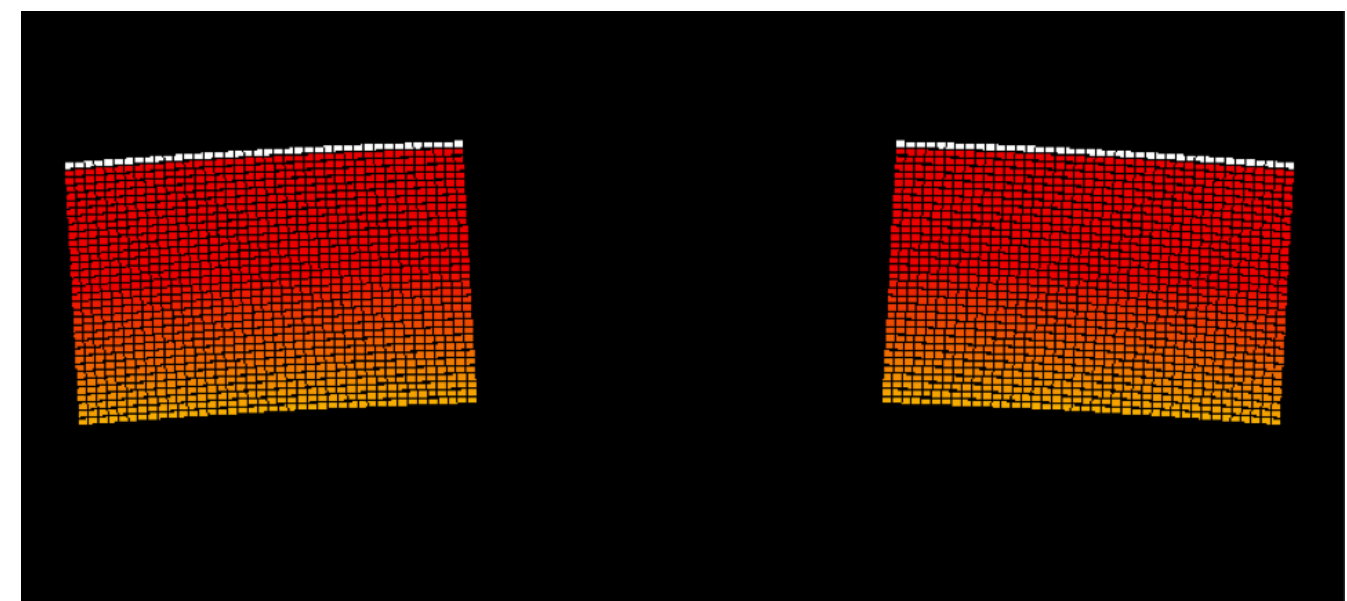

Figure 6. CPF model used to simulate the co-focality of two separated MMs. Two MMs are considered separated by a dark area, the variable shade of red represent the optical path distance variation through the layers. 

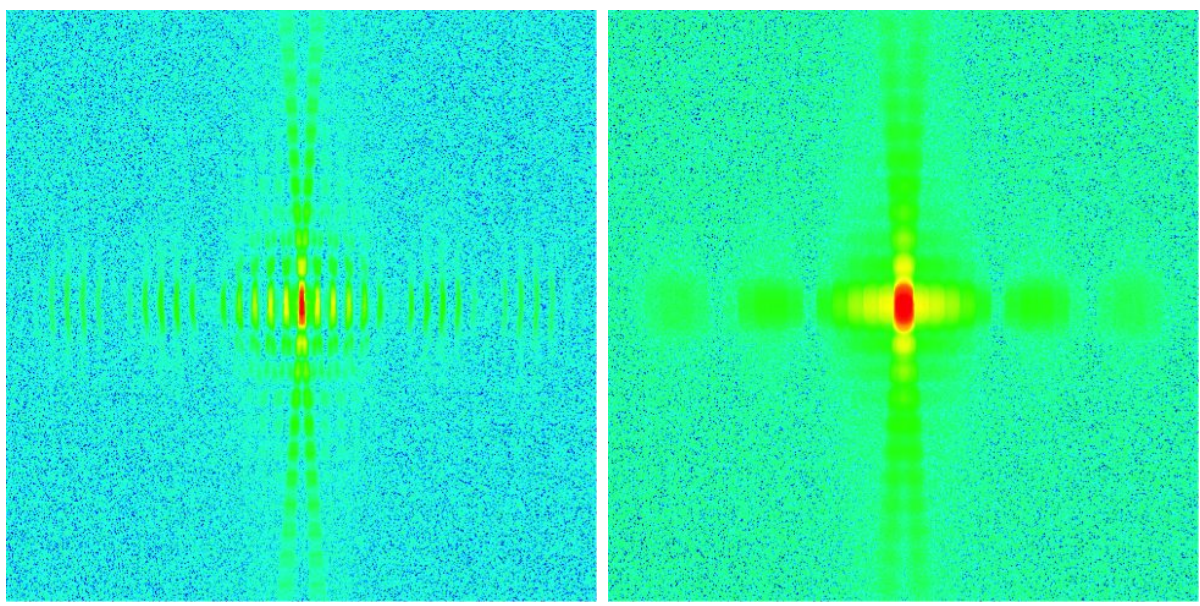

Figure 7. Best-focus configuration of two separated MMs, supposed to have perfect Wolter I surfaces. The simulated detector field is $2 \mathrm{~mm}$ and the pixel size is $2 \mu \mathrm{m}$. Left: PSF of two MMs illuminated by a 0.25 arcsec source. Right: PSF of two MMs illuminated by a 1 arcsec dimension source.

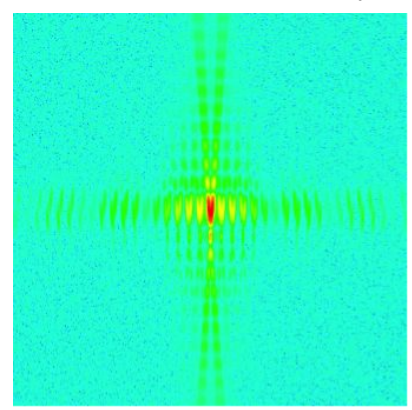

A)

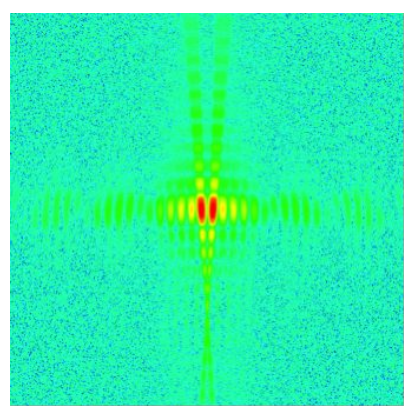

B)

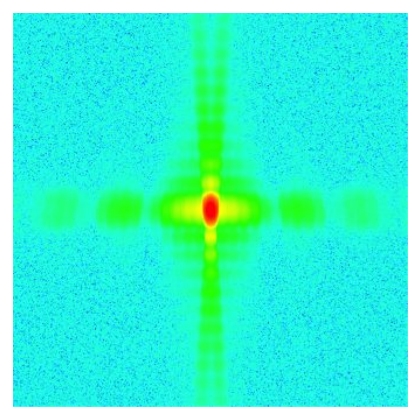

C)

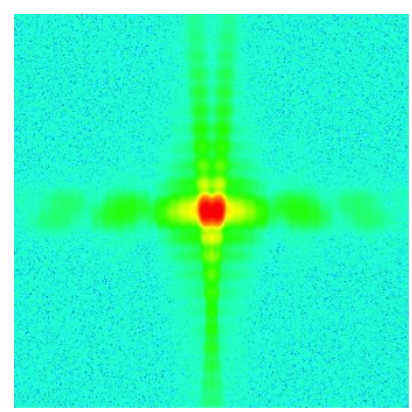

D)

Figure 8. The same configuration shown in Figure 7: moving a $2 \mathrm{~mm}$ wide detector intra-focal by A) $3 \mathrm{~mm}$ and B) $1 \mathrm{~cm}$, considering a source 0.25 arcsec wide. Moving a $2 \mathrm{~mm}$ wide detector intra-focal by C) $3 \mathrm{~mm}$ and D) $1 \mathrm{~cm}$, considering a source 1 arcsec wide.

The detector was simulated as a $2 \mathrm{~mm}$ wide area, with $2 \mu \mathrm{m}$ spatial resolution. The best focus image was calculated at the distance shifted by the finite distance of the source. In this case, the simulated test facility is the X-ray PANTER, ${ }^{10}$ characterized by a source distance of $123.822 \mathrm{~m}$. In this configuration, the best focus for a MM with a focal length of $12 \mathrm{~m}$ results to be shifted by $1.288 \mathrm{~m}$.

To understand how a realistic test could take place we simulated the PSFs moving the detector at intra-focal position, looking for a clear separation of the two foci. In Fig. 8A,B we reported the results obtained for the simulation of a 0.25 arcsec wide source and in Fig. 8C,D for an 1 arcsec wide source at the intra-focal positions of $3 \mathrm{~mm}$ and $1 \mathrm{~cm}$. The separation between the two foci is clearly visible at $3 \mathrm{~mm}$ intra-focal when a small source size is used. Using a larger source, the separation becomes appreciable only defocusing the image by about $1 \mathrm{~cm}$.

\section{MAGNETIC DIVERTER FIELD SIMULATION}

The background caused by soft protons $(\mathrm{E}<100 \mathrm{keV})$ is a major issue in X-ray telescopes, and so is for ATHENA. To avoid the focalization of protons by the optics onto the focal plane instruments without affecting the X-ray flux, a well-known countermeasure is represented by a magnetic field with appropriate orientation and intensity. The magnetic field deviates the particles out of the detector area and so can keep the soft proton background to acceptable levels. In the case of ATHENA, the baseline solution adopted by ESA is represented by an Halbach array of permanent magnets in a pseudo-cylindrical geometry, so that the magnetic field is enhanced in the bore and cancelled outside. The magnetic field confinement, which is essential to avoid perturbating the focal plane detectors, is obtained by rotating the orientation of magnetic dipoles in the array. This approximate geometry 
can be easily obtained by assembling eight trapezoidal bars, forming a closed circuit (Fig. 9). The methodology adopted to model a magnetic field generated by such an assembly of magnetic bars and wedges has been already detailed in a previous paper. ${ }^{8}$

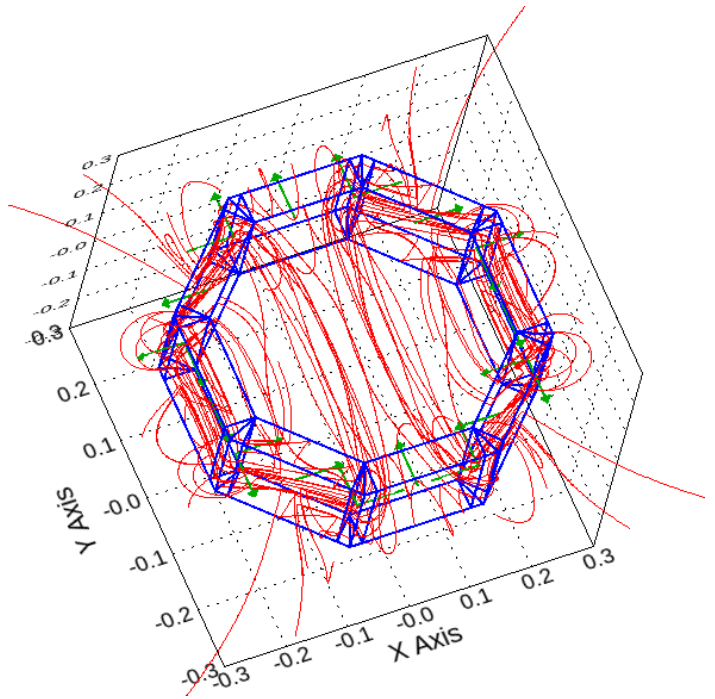

A)

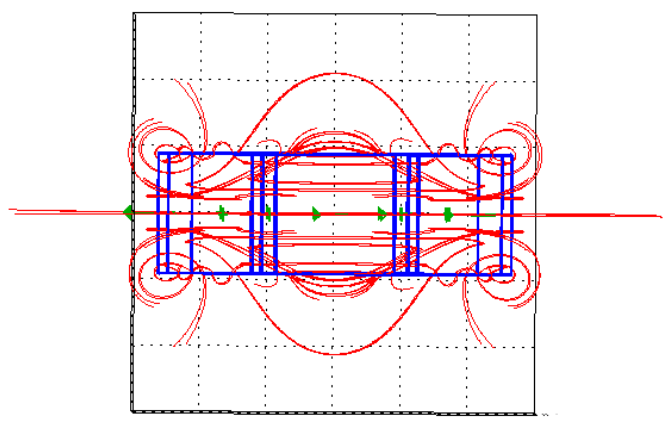

B)

Figure 9. Some simulated magnetic induction field lines, in the updated design of the Halbach array. A) Tilted view. B) Viewed sideways.

We show here an updated sizing of the Halbach array with an enhanced magnetic field, in order to meet not only ATHENA's background specifications, but also the recent simulations on scattering of protons in the ATHENA mirror assembly. ${ }^{12}$ Assuming an ideal configuration with a constant field within the array bore and zero outside, computation shows that in order to deflect even the most laterally-directed proton, at the maximum energy $(76 \mathrm{keV})$ that would make it -after the losses in the filters - fall on the WFI in its sensitivity range, the Halbach array should be $18 \mathrm{~cm}$ long and have a $\sim 500 \mathrm{G}$ field within a $42.6 \mathrm{~cm}$ internal diameter bore. This value assumes the configuration 3 of the optics (Tab. 1), and $1 \mathrm{~m}$ space between the array and the focal plane. We also assume a magnetic dipole density of $1.1 \mathrm{~T}$.

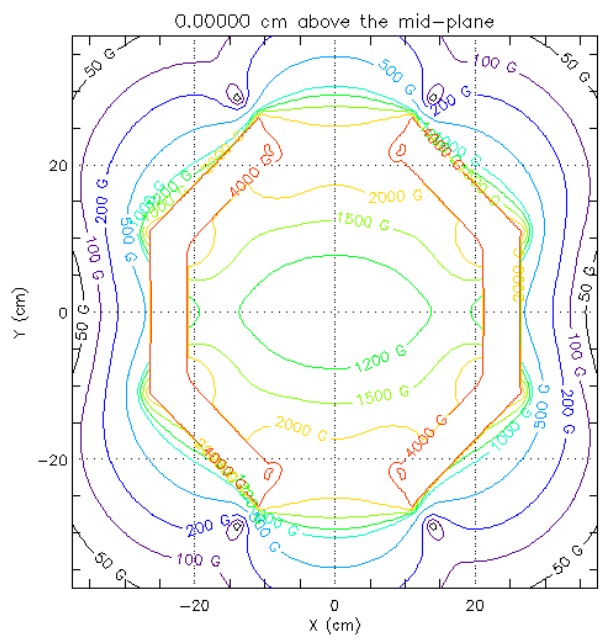

A)

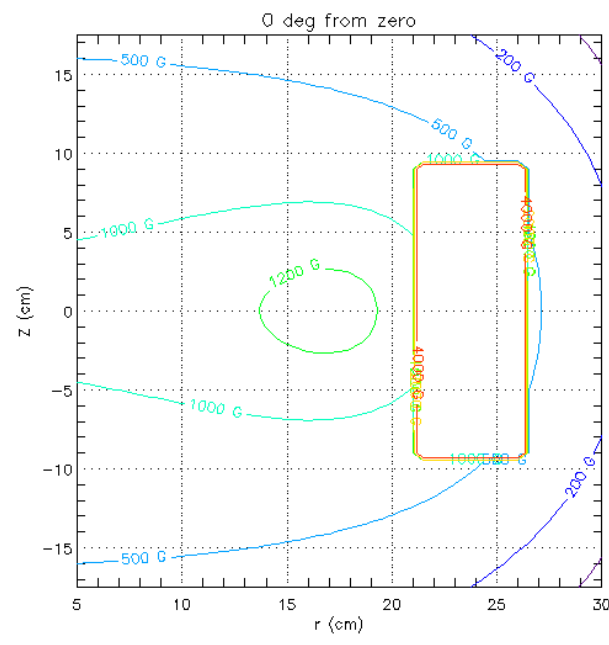

B)

Figure 10. Isocontours of the magnetic induction intensity. A) Median section of the array. B) Radial section. We easily see that the field is above $500 \mathrm{G}$ within all the internal volume of the Halbach array. 
With these assumptions, we could find that the needed level of magnetic field intensity within the bore can be reached easily with $5 \mathrm{~cm}$ radially-thick magnets, and a total mass of the magnetic assembly of $106 \mathrm{~kg}$ (not accounting for the robust structure that will be necessary to safely keep the assembly in place). The magnetic induction intensity is mostly oriented along the $y$-axis (Fig. 9) and exceeds $500 \mathrm{G}$ within all the bore (Fig. 10), while it decays rapidly outside.

Future development of this work will include particle tracing through the simulated magnetic assembly, combining the output of the SPO-particle interaction, as simulated by Geant $4 .{ }^{12}$ The particle tracing routine will allow us to determine the Halbach array efficiency at minimizing the proton background, assuming realistic values for the energy and the angular spread of the oncoming proton beam.

\section{CONCLUSIONS}

In the framework of the SIMPOSiuM project (financed by ESA), INAF-OAB and DTU Space developed a set of software tools to simulate ATHENA optics performances. The simulations cover both domains of geometric and physical optics. In this paper, we reported the results obtained for the optical performance simulations of the new ATHENA design, with reduced maximum diameter of the optics and reflective coating change. We also reported updated results obtained for the ASPHEA project about the alignment of multiple MMs by means of real test facilities and a few simulations of the magnetic field achievable in a Halbach array, generating an effective magnetic field for proton deflection.

\section{ACKNOWLEDGMENTS}

The authors thank ESA for supporting this work with the contracts SIMPOSiuM (contract No. 4000114931) and ASPHEA (contract No. 4000111244).

\section{REFERENCES}

[1] Bavdaz, M., Wille, E., Shortt, B., et al., "The ATHENA optics development," Proc. SPIE 9905, 990527 (2016)

[2] Van Speybroeck, L.P., Chase, R. C., "Design parameters of paraboloid-hyperboloid telescopes for X-ray astronomy," Appl. Opt. 11(2), 440 (1972)

[3] Collon, M. J., Vacanti, G., Günther, R., et al., "Silicon pore optics for the ATHENA telescope," Proc. SPIE 9905, $990528(2016)$

[4] Collon, M., Vacanti, G., Barrière, N., et al., "Development of Athena mirror modules," Proc. SPIE 10399, 103990C (2017)

[5] Della Monica Ferreira, D., Massahi, S., Christensen, F. E., et al., "Design, development, and performance of x-ray mirror coatings for the ATHENA mission," Proc. SPIE 10399, 1039918 (2017)

[6] Della Monica Ferreira, D., Svendsen, S., Massahi, S. et al., "Performance and Stability of Mirror Coatings for the Athena Mission," Proc. SPIE 10699, 106993K (2018)

[7] Spiga, D., Christensen, F., Bavdaz, M., et al, "Simulation and modeling of silicon pore optics for the ATHENA X-ray telescope," Proc. SPIE 9905, $96055 \mathrm{O}$ (2016)

[8] Spiga, D., Della Monica Ferreira, D., Shortt, B., et al., "Optical simulations for design, alignment, and performance prediction of silicon pore optics for the ATHENA x-ray telescope," Proc. SPIE 10399, 103990H (2017)

[9] Valsecchi, G., Marioni, F., Bianucci, G., et al., "Optical integration of SPO mirror modules in the ATHENA telescope," Proc. SPIE 10399, 103990E (2017)

[10] Burwitz, V., Willingale, R., Pellilciari, C., et al., "Testing and calibrating the ATHENA optics at PANTER," SPIE Proc. 10399, 103990O (2017)

[11] Raimondi, L., Spiga, D., "Mirrors for X-ray telescopes: Fresnel diffraction-based computation of point spread functions from metrology," A\&A 573, A22 (2015)

[12] Fioretti, V., Bulgarelli, A., Lotti, S., Macculi, C., et al., "The Geant4 mass model of the ATHENA Silicon Pore Optics and its effect on soft proton scattering," SPIE Proc., this volume (2018) 